TITLE:

\title{
Microscopic hole-transfer efficiency in organic thin-film transistors studied with charge-modulation spectroscopy
}

\section{$\operatorname{AUTHOR(S):~}$}

Miyata, Kiyoshi; Tanaka, Shunsuke; Ishino, Yuuta; Watanabe, Kazuya; Uemura, Takafumi; Takeya, Jun; Sugimoto, Toshiki; Matsumoto, Yoshiyasu

\section{CITATION:}

Miyata, Kiyoshi ... [et al]. Microscopic hole-transfer efficiency in organic thin-film transistors studied with charge-modulation spectroscopy. Physical Review B 2015, 91(19): 195306.

\section{ISSUE DATE:}

2015-05-08

URL:

http://hdl.handle.net/2433/201490

RIGHT:

(C)2015 American Physical Society 


\title{
Microscopic hole-transfer efficiency in organic thin-film transistors studied with charge-modulation spectroscopy
}

\author{
Kiyoshi Miyata, ${ }^{1}$ Shunsuke Tanaka, ${ }^{1}$ Yuuta Ishino, ${ }^{1}$ Kazuya Watanabe, ${ }^{1}$ Takafumi Uemura, ${ }^{2}$ Jun Takeya, ${ }^{2}$ \\ Toshiki Sugimoto, ${ }^{1}$ and Yoshiyasu Matsumoto ${ }^{1, *}$ \\ ${ }^{1}$ Department of Chemistry, Graduate School of Science, Kyoto University, Kyoto 606-8502, Japan \\ ${ }^{2}$ Department of Advanced Materials Science, Graduate School of Frontier Sciences, The University of Tokyo, Chiba 277-8561, Japan
}

(Received 15 November 2014; revised manuscript received 8 April 2015; published 8 May 2015)

\begin{abstract}
While the microscopic transfer properties of carriers are of primary importance for carrier transport of organic semiconductors, the mesoscopic features including the morphologies of grains and the structure of grain boundaries limit the overall carrier transport particularly in polycrystalline organic thin films. Thus the conventional evaluation methods of carrier mobility that rely on macroscopic properties such as $I-V$ curves of devices are not capable to determine carrier transfer probability at the molecular level. Here, we present a method for evaluating the relative strengths of transfer integrals using charge-modulation spectroscopy on thin-film transistors of

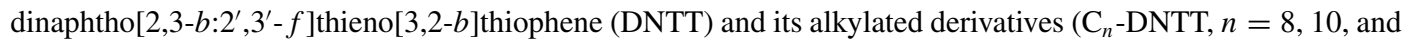
12). The band edges of absorption spectra of holes at around $1.9 \mathrm{eV}$ show bathochromic shifts with increasing length of alkyl chains introduced at both ends of a DNTT chromophore. Applying a two-dimensional model with Holstein-type Hamiltonians to electronic transitions of holes, we have been able to simulate the features of the absorption band edges observed. The simulations indicate that the bathochromic shifts are due to an increase in the transfer integrals of holes with increasing length of alkyl chains. Thus this analysis confirmed that the subtle changes in the mutual orientations between adjacent DNTT chromophores induced by alkyl chains enhance the microscopic hole transfer rate. Although this fastener effect has been suggested by hole mobility measurements by $I-V$ curves, the spectral analysis in this study gives clear evidence of this effect at the molecular level.
\end{abstract}

DOI: 10.1103/PhysRevB.91.195306

\section{INTRODUCTION}

In recent years, there has been an increasing interest in organic semiconductor (OSC)-based devices including lightemitting diodes, thin-film transistors (TFTs), and photovoltaic cells [1]. These devices have been developed with intense effort to realize ultralightweight and low-cost fabrication of flexible electronics. Among them, OSC-TFTs are crucial devices in plastic electronics, where materials with high carrier mobility are essential for fast switching rates [2-4]. While the development of materials with high carrier mobilities for fabrication of superior OSC-TFTs is important in the applied field [5,6], OSC-TFTs bring an interesting question to the scientific community, i.e., the mechanism of carrier transport in OSC.

Intrinsic microscopic carrier transport properties, represented by transfer integrals between adjacent molecules, are of primary importance to realize good carrier mobility of OSC. A key to maximizing the electronic coupling is to introduce a molecular order in the solids of OSC. Among various methods [7], one of the effective ways is introducing alkyl chains to OSC molecules or conjugated polymers to improve "face-to-face" overlap of $\pi$ orbitals, i.e., the fastener effect [8-11]. However, particularly in the case of OSC thin films composed of polycrystallines, the carrier mobility is often strongly influenced by the factors other than intrinsic microscopic properties: the morphologies of grains, the structures of grain boundaries, defects, chemical impurities, etc.

Carrier mobilities of OSCs in crystal forms and thin films are usually evaluated with time-of-flight photocurrent measurements or current-voltage $(I-V)$ characteristics measurements. The mesoscopic morphologies and the extrinsic

\footnotetext{
*matsumoto@kuchem.kyoto-u.ac.jp
}

PACS number(s): 78.66.Qn, 71.38.-k, 72.80.Le, 85.30.Tv

factors such as defects and impurities are often considered to hamper the evaluation of intrinsic carrier mobility; significant variations in the macroscopic transport properties have been found for thermally evaporated OSC thin films under nominally identical conditions $[7,12]$. Thus it is difficult to extract the intrinsic carrier transport properties from the carrier mobility evaluated with the conventional methods.

Spectroscopic probes are capable, in principle, to evaluate intrinsic microscopic carrier transport properties of OSCs. Delocalization of carriers, an important factor for high mobility, has been probed by electron spin resonance (ESR). With the line shape analysis of ESR signals, Marumoto et al. have reported that the wave functions of carriers in pentacene TFTs extend to the order of ten molecules [13]. However, recent works have demonstrated that the linewidths of ESR spectra are not always determined by the extent of delocalization of wave functions, but often governed by motional narrowing in trap-and-release processes of carriers among traps or by carrier hopping across grain boundaries [14-17]. Thus ESR measurements are also strongly influenced by traps and grain boundaries in OSCs.

Charge-modulation spectroscopy (CMS) is another spectroscopic means to gain information of intrinsic carrier mobility of OSC in TFTs under working conditions. In CMS, the absorption spectra of injected carriers are recorded by applying an alternating voltage to the gate electrode of a device; the charge density modulations induced by the alternating voltage allow us to detect carriers with high sensitivity [18-26]. Trapped charges that are not affected by the alternating voltage do not contribute to CM signals. Thus CM signals are mainly contributed by mobile carriers in TFTs. Thus CMS is an excellent method of identifying the absorption spectra of mobile carriers. Moreover, because the absorption spectra 
(a)

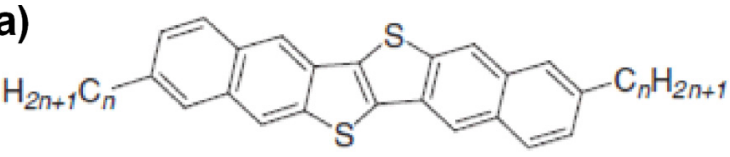

$\mathrm{C}_{n}$-DNTT $(n=0,8,10,12)$

(b)

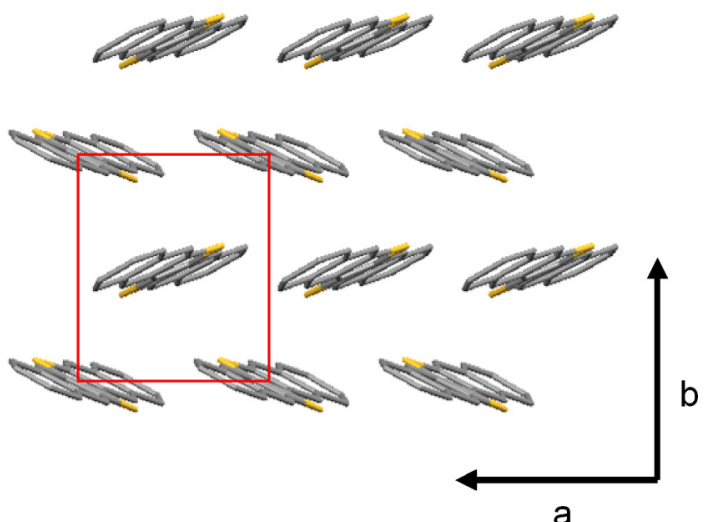

FIG. 1. (Color online) (a) Molecular structure of $\mathrm{C}_{n}$-DNTT $(n=$ $0,8,10$, and 12). (b) Herringbone structure of DNTT in $a b$ plane. A centered rectangular unit cell containing two DNTT molecules is depicted with solid red line.

of carriers are sensitive to the transfer integrals of charges, it is possible to obtain the information of charge transfer characteristics of OSCs at the molecular level. However, in previous CMS studies on TFTs, the band shapes of CM spectra have not been analyzed in detail; at most, the transition energies of cations of a monomer or a dimer of OSC molecules have been estimated with quantum-mechanical calculations for assigning the origins of absorption bands observed [21,26]. To deduce the information of intrinsic properties from the band shapes of CM spectra, we need to analyze them by considering the delocalized nature of charges and electron-phonon couplings associated with electronic excitations. Although the Holstein-type model [27] has been successfully applied to analyze the absorption and emission spectra of aggregated neutral molecules to extract the microscopic properties of excitons $[28,29]$, it has not been applied to the analysis of CM spectra except for the infrared absorption bands of conjugated polymer semiconductors [30].

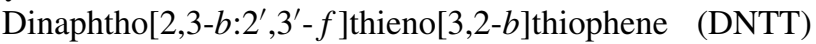
[31] and its alkylated derivatives $\left(\mathrm{C}_{n}\right.$-DNTT, $n=8,10$, and 12) are the choice of molecules in this study; the molecular structure of $\mathrm{C}_{n}$-DNTT and the characteristic herringbone structure in the $a b$ plane of a DNTT crystal are depicted in Fig. 1. It has been reported that the hole mobilities of these compounds evaluated from $I-V$ curves depend on the length of alkyl-chains introduced at both ends of a DNTT chromophore $[32,33]$; this could be understood by the fastener effect where the orbital overlap in the $\pi$ stacking of DNTT chromophores is improved through van der Waals interactions of alkyl chains. However, this assertion has to be tested in terms of microscopic properties of carrier transfer, because the hole mobilities obtained by $I-V$ curves may be limited by the mesoscopic morphologies of the thin films.
In this paper, we report measurements that provide both macroscopic and microscopic information regarding the hole mobilities in TFTs of these compounds. We have performed, on the one hand, $I-V$ characteristics measurements to evaluate the macroscopic hole mobilities as a function of alkylchain length and observed thin film morphologies using an atomic force microscope (AFM). On the other hand, we have measured the absorption spectra of holes by CMS with the same devices used in the $I-V$ curve measurements and analyzed the spectra by applying a two-dimensional model with the Holstein-type Hamiltonians to evaluate microscopic hole transfer integrals. We demonstrate that the spectral features at the band edges of absorption spectra of holes are directly linked to hole transfer integrals of OSCs. Thus the alkyl-chain-length $(n)$ dependence of hole transfer integrals from CM spectra provides a critical test for the fastener effect at the molecular level. In addition, the comparison with the $n$ dependence of hole mobility from $I$ - $V$ curves allows us to determine which factor limits hole mobility: microscopic or mesoscopic structure.

\section{EXPERIMENTAL SECTION}

We fabricated bottom-gate top-contact TFTs of DNTT and $\mathrm{C}_{n}$-DNTT $(n=8,10$, and 12). All materials were used as received (Nippon Kayaku). DNTT or $\mathrm{C}_{n}$-DNTT was vapor deposited on a $\mathrm{SiO}_{2}$ (100-nm-thick)/n-Si substrate covered with a self-assembled monolayer of dodecyltriethoxysilane at room temperature for DNTT and at $80^{\circ} \mathrm{C}$ for $\mathrm{C}_{n}$-DNTT, followed by $\mathrm{Au}$ vapor deposition for fabricating source and drain electrodes. With an AFM, we observed the morphologies and the thickness of the thin films. The channel length and width of devices were both $1 \mathrm{~mm}$. The carrier mobility of the TFTs was estimated from $I-V$ characteristics measured in the range of $-20<V_{\mathrm{g}}<20 \mathrm{~V}$ at $V_{\mathrm{d}}=-1 \mathrm{~V}\left(V_{\mathrm{g}}\right.$ : gate voltage, $V_{\mathrm{d}}$ : drain voltage). For the measurements of absorption spectra of cations of the OSC molecules, DNTT and $\mathrm{C}_{10}$-DNTT were dissolved in ortho-dichlorobenzene $\left(<3 \times 10^{-6} \mathrm{M}\right)$ separately and oxidized with $\mathrm{FeCl}_{3}$. Thin films of DNTT (50-nm thick) and $\mathrm{C}_{10}$-DNTT (20-nm thick) were fabricated by vapor deposition for the measurements of absorption spectra of aggregated neutral molecules.

For CMS measurements, the reflected light of a halogen lamp from the OSC thin film of the device was dispersed in a polychromator (SOLAR TII) and detected with a chargecoupled device camera (Andor technology). CM spectra were measured by modulating $V_{\mathrm{g}}$ at $1 \mathrm{~Hz}$ with a rectangular wave that switched alternately the device between charge accumulation $\left(V_{\mathrm{g}}=-12 \mathrm{~V}\right)$ and depletion $\left(V_{\mathrm{g}} \geqslant+5 \mathrm{~V}\right)$ conditions, while $V_{\mathrm{d}}$ was kept at $-1 \mathrm{~V}$. We defined the $\mathrm{CM}$ signal, i.e., the modulation depth in reflectance $R$, as $\Delta R / R=\left(I_{\mathrm{a}}-I_{\mathrm{d}}\right) / I_{\mathrm{d}}$, where $I_{\mathrm{a}}$ and $I_{\mathrm{d}}$ are the reflected light intensities under the accumulation and depletion conditions, respectively.

\section{RESULTS AND DISCUSSION}

\section{A. Macroscopic hole mobility}

The hole mobilities of TFTs estimated from $I-V$ characteristics are summarized in Table I. The DNTT TFT shows the lowest hole mobility among the TFTs tested. Although 
TABLE I. Hole mobilities of TFTs of DNTT and $\mathrm{C}_{n}$-DNTT obtained from $I-V$ characteristics of TFTs. (See Fig. S1 in Ref. [36] for the raw data of $I-V$ characteristics.)

\begin{tabular}{lc}
\hline \hline Material & Hole mobility $\left(\mathrm{cm}^{2} \mathrm{~V}^{-1} \mathrm{~s}^{-1}\right)$ \\
\hline DNTT & 1.2 \\
$\mathrm{C}_{8}$-DNTT & 1.7 \\
$\mathrm{C}_{10}$-DNTT & 4.9 \\
$\mathrm{C}_{12}$-DNTT & 2.8 \\
\hline \hline
\end{tabular}

introducing alkyl chains to DNTT enhances the hole mobility, the mobility does not necessarily correlate well with the alkyl chain length; the hole mobility increases as the chain length is changed from $n=8$ to 10 , but decreases at the longer chain of $n=12$. This $n$ dependence of hole mobility is in good agreement with the previous work [32].

\section{B. Absorption spectra of cations}

As shown in Fig. 2, the absorption spectra of cations of DNTT and $\mathrm{C}_{10}$-DNTT dissolved in ortho-dichlorobenzene appear in an energy range lower than that of the HOMOLUMO transition of neutral molecules located at around $3.1 \mathrm{eV}$. The spectral features of DNTT and $\mathrm{C}_{10}$-DNTT cations are almost identical, indicating that the DNTT chromophore is responsible for the absorption bands. Two absorption bands contribute to the absorption spectra of cations in this energy range: the broad featureless band within $1.6-2.0 \mathrm{eV}$ and the band within $2.0-2.6 \mathrm{eV}$ with a pronounced vibrational progression with an energy interval of $0.18 \mathrm{eV}$ corresponding to $\mathrm{C}-\mathrm{C}$ stretching modes. The relative peak intensities of the vibrational progression are well reproduced by a Poisson distribution $I_{m}=g^{2} / m$ ! with an electron-phonon coupling constant of $g=0.74$.

We calculated the electronic transition energies of an isolated DNTT cation with a time-dependent density functional theory (TD-DFT) after optimizing the geometry of DNTT

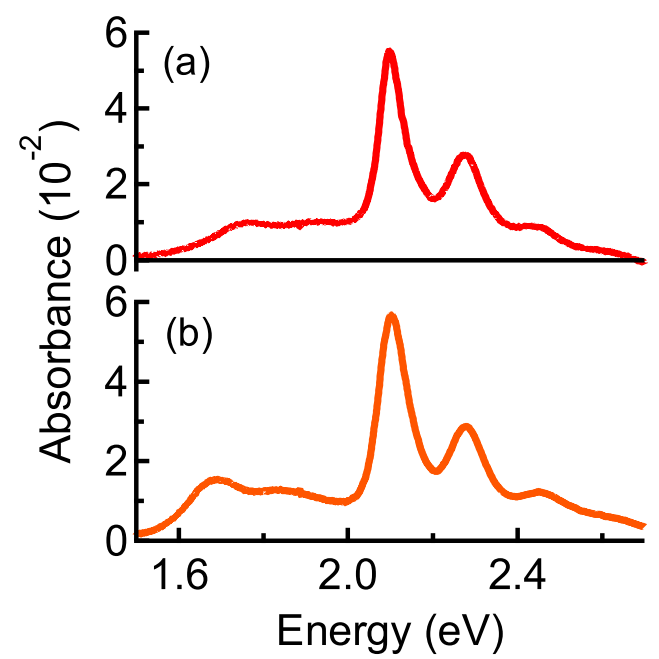

FIG. 2. (Color online) Absorption spectra of cations of (a) DNTT and (b) $\mathrm{C}_{10}$-DNTT dissolved in ortho-dichlorobenzene. A fraction of dissolved neutral molecules was oxidized with $\mathrm{FeCl}_{3}$ saturated in the solution.

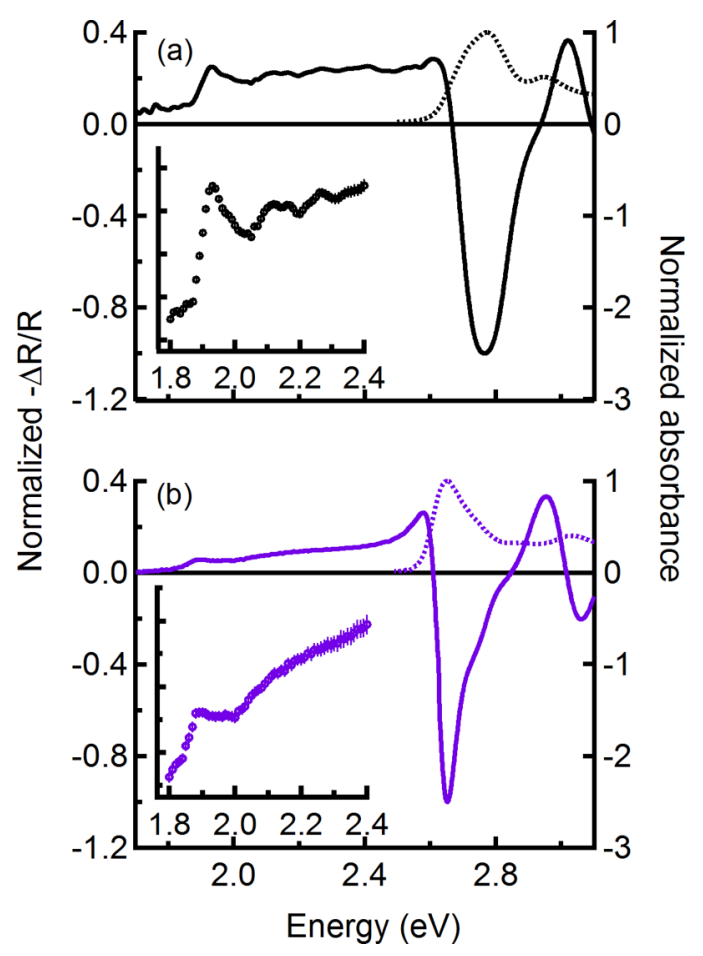

FIG. 3. (Color online) CM spectra of TFTs (solid lines) and absorption spectra of thin films (broken lines) of (a) DNTT and (b) $\mathrm{C}_{10}$-DNTT. The insets are the absorption bands near the band edge where the amplitudes are expanded by a factor of (a) 2.5 and (b) 4 , respectively.

cation in the ground state. The basis set of $6-31+(\mathrm{d}, \mathrm{p})$ and the hybrid functional of unrestricted B3LYP in the GAUSSIAN 09 package [34] were used. On the basis of the calculations, the absorption band in $1.6-2.0 \mathrm{eV}$ was assigned to the transition from HOMO-3 to singly occupied molecular orbital (SOMO), and the absorption band in $2.0-2.6 \mathrm{eV}$ to the transition from SOMO to LUMO.

\section{Charge-modulation spectra}

CM spectra of DNTT and $\mathrm{C}_{10}$-DNTT are shown in Fig. 3 together with the absorption spectra of thin films of these compounds. Both the spectra show similar features: the absorption signals in 1.8-2.4 eV and bleaching in the energy range of the absorption bands of neutral molecules. The bleaching spectral profiles reflect on those of absorption bands of neutral molecules; the band of DNTT is broader than that of $\mathrm{C}_{10}$-DNTT. Thus the bleaching is clearly induced by reduction of the number of neutral molecules caused by hole injection. The absorption bands in $1.8-2.4 \mathrm{eV}$ are due to injected holes. We focus in this paper on the transition from SOMO to LUMO in the $\mathrm{CM}$ spectra, corresponding to the absorption band of cations in solution in $2.0-2.6 \mathrm{eV}$, because the transfer integrals of charge for these states are readily available, which allows us to perform spectral simulations as described in Sec. IIIE. We attributed the absorption band in $1.8-2.4 \mathrm{eV}$ with a shoulder observed at around $1.9 \mathrm{eV}$ in the $\mathrm{CM}$ spectra to the transition from SOMO to LUMO to the following reasons: (1) the absorption bands of cations (Fig. 2) in 2.0-2.6 eV (SOMO $\rightarrow$ LUMO) are stronger than those in 1.6-2.0 eV (HOMO-3 $\rightarrow$ SOMO) 
by a factor of 3-5; (2) if the band of cation in 1.6-2.0 eV were responsible for the band of $\mathrm{CM}$ spectra in $1.8-2.6 \mathrm{eV}$, a more intense band would be observed in the higher energy of CM spectra around $2.3 \mathrm{eV}$ due to the cation band around $2.1 \mathrm{eV}$, but there is no such intense band in the CM spectra; (3) the absorption band of holes in DNTT in 1.8-2.4 eV shows a vibrational progression similar to that of DNTT cation in $2.1-2.6 \mathrm{eV}$ whereas the absorption band of cation in $1.6-2.0 \mathrm{eV}$ does not; (4) the shoulder at around $1.9 \mathrm{eV}$ in the CM spectra is redshifted by about $0.2 \mathrm{eV}$ from the bands of cations around $2.1 \mathrm{eV}$, which is compatible to the shift of the absorption band of neutral species in the thin film from those in solution.

Note that the CM spectra above $2.8 \mathrm{eV}$ show derivativelike features. The strong spectral modulations could be due to electroabsorption associated with Stark shifts of the absorption band of neutral species induced by applied electric field. In contrast, the absorption band of hole in $1.8-2.4 \mathrm{eV}$ is free from this effect, because no electronic transitions of neutral species overlap with the hole absorption band. The hole absorption band could depend on the gate voltage through a strong local electric field of surrounding holes injected [35]. However, we confirmed that the spectral features of the hole absorption band do not depend on the applied gate voltage (see Fig. S3 in Ref. [36]). This indicates that the density of injected holes is small so that the local field of a hole does not influence the electronic structure of the other hole.

Although the energy ranges of the absorption bands of holes are similar, the spectral feature of DNTT is different from that of $\mathrm{C}_{10}$-DNTT: DNTT shows a relatively sharp peak at $1.93 \mathrm{eV}$ accompanied with a faint vibrational progression of $0.18 \mathrm{eV}$, a similar energy interval of that of DNTT cations (Fig. 2), but $\mathrm{C}_{10}$-DNTT shows a smooth monotonically increasing band shape with a shoulder at $1.88 \mathrm{eV}$, with no appreciable vibrational progression on the absorption spectrum.

Clear chain-length dependence was observed in the holeinduced absorption bands. As in Fig. 4, the absorption band edges of holes of $\mathrm{C}_{n}$-DNTT show bathochromic shifts with increasing alkyl-chain length; the energy of the shoulder in the band edge is shifted from $1.93,1.90,1.88$, to $1.86 \mathrm{eV}$ for $n=0$, 8,10 , and 12 , respectively. The similar bathochromic shifts of the lowest absorption band of the neutral species in thin films were also observed (Fig. S2 in Ref. [36]). These shifts indicate that subtle differences in the assembled structure of molecules, particularly DNTT chromophores, induced by alkyl chains affect the electronic states of both holes and neutral molecules in the thin films.

\section{Hole transfer versus intramolecular vibronic coupling}

The clear vibrational progression in the electronic absorption band of isolated molecules originates in intramolecular vibronic coupling. The progression is often blurred when molecules are aggregated because of line broadening by static and dynamic disorder in addition to the effect of intermolecular electronic coupling. Assuming that the line broadening is dominated over the intermolecular coupling effect, we estimated the full width at half maximum of the band in the CM spectra to be at most $0.08 \mathrm{eV}$ by fitting the lower edge of the spectrum of $\mathrm{C}_{8}$-DNTT with a Gaussian function having a peak at $1.9 \mathrm{eV}$. This width is slightly larger than the width of

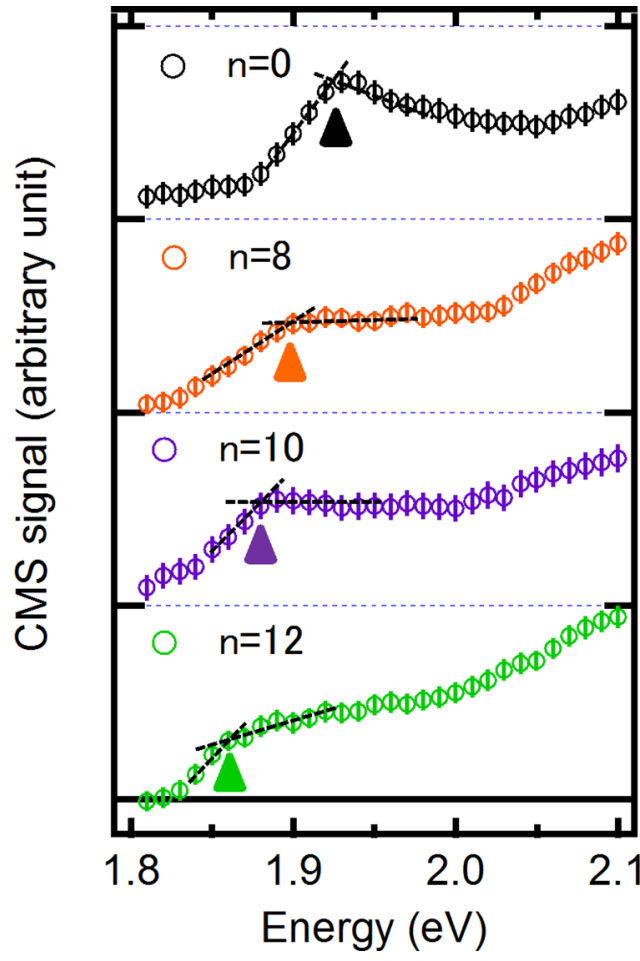

FIG. 4. (Color online) Absorption band edge structures in the CM spectra of DNTT $(n=0)$ and $\mathrm{C}_{n}$-DNTT TFTs $(n=8,10$, and 12) with error bars. Arrows are the guide to the eyes for the position of shoulders.

the cation absorption spectra, $0.05 \mathrm{eV}$. Thus, although the line broadening partially blurs the vibrational progression, it cannot smooth it out completely. Moreover, the gradual increase in intensity with photon energy observed in Figs. 3 and 4 is not reproduced by inclusion of the line broadening only. Therefore the loss of vibrational progression in the films of alkylated DNTTs is mainly attributed to intermolecular coupling.

In the limit of weak intermolecular coupling, the vibrational progression can still exist even in aggregated molecules. In contrast, the vibrational progression disappears, whereas excitonic features appear in the limit of strong intermolecular coupling. The transition of spectral features between these limits has been well described by the Holstein model for Frenkel excitons [37,38]. The analysis of spectral features of molecular aggregates provides the parameters relevant to exciton dynamics including the intermolecular electronic coupling strength and the exciton coherence size [39].

Spectral features of absorption bands of holes injected in molecular aggregates also provide information of intermolecular couplings and hole transfer rates at the molecular level. Two time scales are relevant to the spectral features: the period of an intramolecular vibrational mode $\left(\tau_{v}\right)$ responsible for the vibrational progression and the characteristic time for hole transfer in the electronically excited state $\left(\tau_{e}\right)$. In view of the time-domain picture, a clear vibrational progression appears if $\tau_{v} \ll \tau_{e}$, while vibrational progression disappears if $\tau_{v} \gg \tau_{e}$. Thus the intramolecular vibration can be an internal clock for excited hole transfer dynamics. In this context, we can judge qualitatively that the excited hole transfer rate in DNTT is slower than that in alkylated-DNTTs, because the hole 
absorption band of DNTT shows the faint vibrational progression but those of alkylated-DNTTs do not. This argument gives a qualitative explanation of the faster excited hole transfer and hence higher hole mobility of alkylated-DNTTs than those of DNTT. However, it is too crude to explain the relation between the absorption-band-edge shift and hole mobility; both of them depend on the alkyl-chain length in different ways. Thus we need to elaborate the spectral features of absorption bands of holes in more details by means of theoretical simulations, which we describe in the next section.

\section{E. Simulation of absorption spectra of holes}

\section{Model Hamiltonian and basis set}

Here, we describe a model based on the exciton theory with a Holstein-type Hamiltonian $[37,39,40]$ for simulating the spectral features of absorption bands of holes observed in the CMS measurements. Note that we need to take into account the dispersion of holes in the ground state explicitly here (see Ref. [36], Secs. VIII and IX). This is in sharp contrast to the standard exciton model for the absorption bands of neutral species, where a vibrationless electronic ground state is taken as an initial state of the electronic transition. The model consists of an infinite two-dimensional (2D) molecular array with two molecules per unit cell; this mimics the herringbone crystalline structure of DNTT [Fig. 1(b)]. Modeling the electronic structure and carrier dynamics of the current systems with this $2 \mathrm{D}$ array model is reasonable, because carriers are injected and transported mostly in the single layer of molecules at the OSC/insulator interface in the TFT. Molecules in the 2D array are labeled by two indices: the vector $\mathbf{n}$ for labeling a unit cell and the sublattice index $\sigma$ for labeling a molecule in the unit cell. On the basis of the TD-DFT calculations (Sec. III B), we assign the absorption band of holes to the transition of SOMO $\rightarrow$ LUMO, whose transition dipole moment is perpendicular to the herringbone sheet.

The one-exciton Hamiltonian of holes in the electronically excited state $H$ is given below in the manner of the conventional Holstein-type model. We assume that the electronic transition is coupled to only one intramolecular vibrational mode of a frequency $\omega_{v}$ and the potential curves with respect to this mode in the ground and electronically excited states are all harmonic:

$$
\begin{aligned}
H & =H_{\mathrm{e}}+H_{\mathrm{ph}}+H_{\mathrm{e}-\mathrm{ph}}, \\
H_{\mathrm{e}} & =\sum_{\mathbf{n} \sigma} E_{0} a_{\mathbf{n} \sigma}^{\dagger} a_{\mathbf{n} \sigma}+\sum_{\mathbf{n} \sigma, \mathbf{m} \sigma^{\prime}}\left(1-\delta_{n m} \delta_{\sigma \sigma^{\prime}}\right) J_{\mathbf{n} \sigma \mathbf{m} \sigma^{\prime}} a_{\mathbf{n} \sigma}^{\dagger} a_{\mathbf{m} \sigma^{\prime}} \\
H_{\mathrm{ph}} & =\sum_{\mathbf{n} \sigma} \hbar \omega_{v} b_{\mathbf{n} \sigma}^{\dagger} b_{\mathbf{n} \sigma}, \\
H_{\mathrm{e}-\mathrm{ph}} & =\sum_{\mathbf{n} \sigma} \hbar \omega_{v} g_{\mathrm{e}} a_{\mathbf{n} \sigma}^{\dagger} a_{\mathbf{n} \sigma}\left(b_{\mathbf{n} \sigma}^{\dagger}+b_{\mathbf{n} \sigma}\right) .
\end{aligned}
$$

Here, $E_{0}$ is the energy of a dipole-allowed electronically excited hole state; $a_{\mathbf{n} \sigma}^{\dagger}\left(a_{\mathbf{n} \sigma}\right)$ is the creation (annihilation) operator for an excited hole state localized at a molecule $\mathbf{n} \sigma$, and $b_{\mathbf{n} \sigma}^{\dagger}\left(b_{\mathbf{n} \sigma}\right)$ is the creation (annihilation) operator for a coupled phonon at the same molecule; $J_{\mathbf{n} \sigma \mathbf{m} \sigma^{\prime}}$ is the intermolecular electronic coupling between two molecules located at $\mathbf{n} \sigma$ and $\mathbf{m} \sigma^{\prime}$; and $g_{\mathrm{e}}$ is the electron-phonon coupling constant.

Considering the polaronic energy structure in the ground state of a hole, we constructed the Hamiltonian of holes in the ground state in a similar way with the electronic couplings $J_{\mathbf{n} \sigma \mathbf{m} \sigma^{\prime}}^{\prime}$ instead of $J_{\mathbf{n} \sigma \mathbf{m} \sigma^{\prime}}$ (see Ref. [36], Sec. VII ).

In the case of materials composed of neutral organic molecules, electronic excitations result in the formation of Frenkel excitonic polarons: a Frenkel exciton surrounded by vibrationally (but not electronically) excited molecules [39]. Similar collective excitations can occur in the case of electronic excitations of holes. Thus it is appropriate to describe the collective excitations with many-particle states. In this work, we represent the Hamiltonian with a delocalized basis set under the two-particle approximation (TPA) [40,41]: a single vibronic excitation plus a vibronic/vibrational pair excitation (see Ref. [36], Sec. IV). Because three-particle states contribute little to the absorption and emission [40,42], TPA is appropriate for obtaining the optical response in organic aggregates.

\section{Absorption spectra of holes}

Electronic absorption spectra of holes $A_{\text {hole }}(E)$ are calculated with

$$
\begin{aligned}
A_{\text {hole }}(E) \propto & \int_{\mathbf{k}} \sum_{j, j^{\prime}} p_{j^{\prime}}\left|\left\langle\Psi_{j^{\prime}, \mathbf{k}}^{\mathrm{c}}|\mu| \Psi_{j, \mathbf{k}}^{\mathrm{e}}\right)\right|^{2} \\
& \times \Gamma\left(E-\left(E_{j, \mathbf{k}}-E_{j^{\prime}, \mathbf{k}}\right)\right) \mathrm{d} \mathbf{k},
\end{aligned}
$$

where $\left|\Psi_{j^{\prime}, \mathbf{k}}^{\mathrm{c}}\right\rangle$ and $\left|\Psi_{j, \mathbf{k}}^{\mathrm{e}}\right\rangle$ are the $j^{\prime}$ th initial and $j$ th final electronic states of holes, respectively, $E_{j^{\prime}, \mathbf{k}}$ and $E_{j, \mathbf{k}}$ are the eigenenergies of $\left|\Psi_{j^{\prime}, \mathbf{k}}^{\mathrm{c}}\right\rangle$ and $\left|\Psi_{j, \mathbf{k}}^{\mathrm{e}}\right\rangle$, respectively, $\mu$ is the dipole moment operator, $p_{j^{\prime}}$ is the occupation probability for the $j^{\prime}$ th initial state, and $\Gamma(E)$ is the line-shape function. Taking account of the electronic coupling for holes reported for DNTT crystals [43], we found that the lowest holes state is at $\mathbf{k}_{0}=\left(k_{\mathrm{x}}, k_{\mathrm{y}}\right)=(0,0)$, where $k_{\mathrm{x}}$ and $k_{\mathrm{y}}$ are wave numbers along $a$ and $b$ axes of a DNTT crystal, respectively, and the energy difference between the lowest and the second lowest states in the stack at $\mathbf{k}_{0}$ is $167 \mathrm{meV}$ (see Ref. [36], Sec. VIII). Thus we have taken into account the transition only from the lowest state $\left(j^{\prime}=0\right)$ at $\mathbf{k}_{0}$. Consequently, $A_{\text {hole }}(E)$ can be described as

$$
\begin{aligned}
A_{\text {hole }}(E) \propto & \sum_{j} \mid\left(\sum_{\sigma, \tilde{u}, \tilde{v}} d_{\sigma \tilde{u}}^{0, \mathbf{k}_{0}} c_{\sigma \tilde{v}}^{j, \mathbf{k}_{0}}\langle\mathrm{c} ; \chi(\tilde{u}) \mid \mathrm{e} ; \chi(\tilde{v})\rangle\right. \\
& +\sum_{\sigma, \tilde{u}, \tilde{v} \mathbf{R}, \sigma^{\prime}, u, v} \sum_{\sigma \tilde{u}, \mathbf{R} \sigma^{\prime} u} c_{\sigma \tilde{v}, \mathbf{R} \sigma^{\prime} v}^{j, \mathbf{k}_{0}} \\
& \times\langle\mathrm{c} ; \chi(\tilde{u}) \mid \mathrm{e} ; \chi(\tilde{v})\rangle\langle\mathrm{g} ; \xi(u) \mid \mathrm{g} ; \eta(v)\rangle)\left.\right|^{2} \\
& \times \Gamma\left(E-E_{j 0}\right),
\end{aligned}
$$

where $u$ and $v$ are the quanta of vibrational modes in the neutral ground state; $\tilde{u}$ and $\tilde{v}$ are those in the ground and excited states of hole, respectively; $\langle\mathrm{c} ; \chi(\tilde{u}) \mid \mathrm{e} ; \chi(\tilde{v})\rangle$ is the Franck-Condon integral between vibrational wave functions in a hole ground state $|\mathrm{c} ; \chi(\tilde{u})\rangle$ and a hole excited state $|\mathrm{e} ; \chi(\tilde{v})\rangle$; 
$\langle\mathrm{g} ; \xi(u) \mid \mathrm{g} ; \eta(v)\rangle$ is the overlap integral between the vibrational wave functions of modes: $\xi$ and $\eta$ in the ground state of neutral molecules; $E_{j 0}$ is the transition energy from the initial state to the $j$ th excited state at $\mathbf{k}_{0}\left(E_{j 0}=E_{j, \mathbf{k}_{0}}-E_{0, \mathbf{k}_{0}}\right) ; c_{\sigma \tilde{v}}^{j, \mathbf{k}}$ and $c_{\sigma \tilde{v}, \mathbf{R} \sigma^{\prime} v}^{j, \mathbf{k}}$ are the coefficients for one- and two-particle states in the excited state of hole, respectively; $d_{\sigma \tilde{u}}^{j, \mathbf{k}}$ and $d_{\sigma \tilde{u}, \mathbf{R} \sigma^{\prime} u}^{j, \mathbf{k}}$ are those of one- and two-particle states of delocalized holes in the ground state, respectively. The coefficients are evaluated numerically by diagonalization of the Hamiltonian for the ground and excited states of holes with the basis sets of oneand two-particle states in the momentum space. The matrix elements of Hamiltonian used for diagonalization can be found in Ref. [36], Sec. V.

In general, the vibrational level structures of hole states in the electronically ground and excited states depend on the vibrational coordinate and its displacement associated with the hole transfer. We examined two extreme cases: (i) the vibrational coordinate displaced by hole transfer in the ground state $\left(Q_{\xi}\right)$ and the one displaced by hole transfer in the electronically excited state $\left(Q_{\eta}\right)$ are identical; and (ii) the two coordinates $Q_{\xi}$ and $Q_{\eta}$ are orthogonal. We will show that case (ii) is more likely because the spectral features of CM spectra observed can be simulated more satisfactorily than case (i). Thus we focus on the simulation in the case (ii) in this paper. The simulation in case (i) can be found in Ref. [36] (see Sec. X).

Because the terms $\langle\mathrm{g} ; \xi(u) \mid \mathrm{g} ; \eta(v)\rangle$ is zero in the case of (ii), Eq. (3) can be simplified as

$A_{\text {hole }}(E) \propto \sum_{j}\left|\sum_{\sigma, \tilde{u}, \tilde{v}} d_{\sigma \tilde{u}}^{0, \mathbf{k}_{0}} c_{\sigma \tilde{v}}^{j, \mathbf{k}_{0}}\langle\mathrm{c} ; \chi(\tilde{u}) \mid \mathrm{e} ; \chi(\tilde{v})\rangle\right|^{2} \Gamma\left(E-E_{j 0}\right)$.

In the numerical evaluations of absorption spectra using Eq. (4) as a function of intermolecular electronic coupling strength, we fixed the maximum vibrational quanta of $\tilde{u}$ and $\tilde{v}$ to be 3 and included 49 unit cells (98 molecules) for describing phonon-dressed excited states. The frequency of the vibrational mode of interest $\omega_{v}$ was set to be $\hbar \omega_{v}=0.18 \mathrm{eV}$. The electron-phonon coupling constant was estimated to be 0.74 by fitting of the measured absorption spectrum of DNTT cations in the ortho-dichlorobenzene solution (Fig. 2) and fixed at this value throughout the simulations. The line shape of individual transition was assumed to be Gaussian $\Gamma(E)=\exp \left(-E^{2} / \gamma^{2}\right)$ with $\gamma=0.005 \mathrm{eV}$.

\section{F. Origin of intermolecular coupling}

The spectral features of absorption bands of holes are mainly governed by electron-phonon couplings and intermolecular electronic coupling constants both in the ground and excited states of hole. Because the one-electron transfer integral between HOMOs $t_{\mathrm{h}}$ dominates in the energy structure of the initial states (see Ref. [36], Sec. VII), we assume that the electronic coupling in the ground state is approximated as $J^{\prime} \sim t_{\mathrm{h}}$. In contrast, the intermolecular electronic coupling constant in the excited state of hole $J$ is described as

$$
J=\left\langle\mathbf{A} \Psi_{\mathbf{a}}^{\mathrm{e}} \Psi_{\mathbf{b}}^{\mathrm{g}}\left|H_{\mathrm{e}}\right| \mathbf{A} \Psi_{\mathbf{a}}^{\mathrm{g}} \Psi_{\mathbf{b}}^{\mathrm{e}}\right\rangle,
$$

(a)
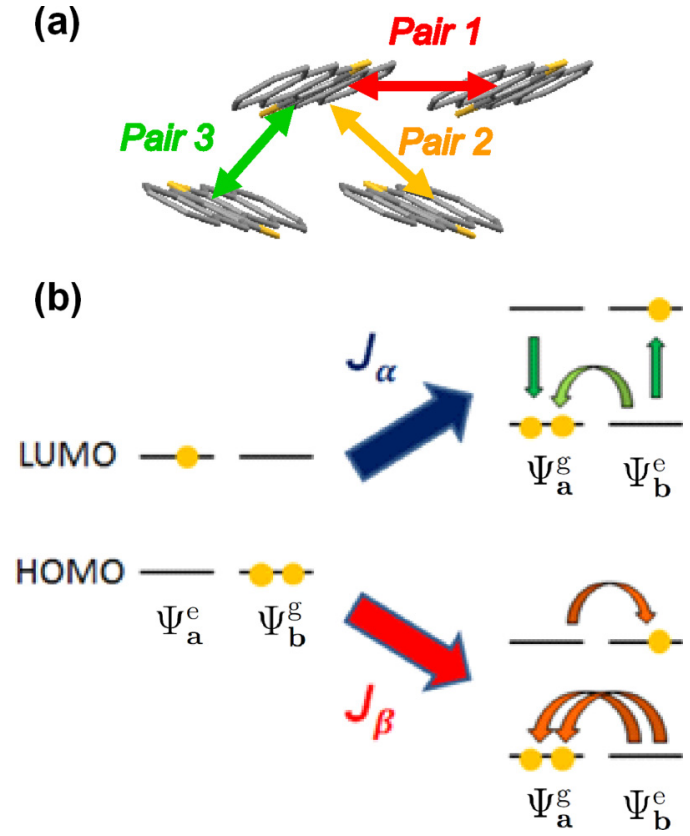

FIG. 5. (Color online) (a) Three different neighboring pairs in a DNTT crystal. (b) Schematic representations of transitions from $\left|\Psi_{\mathbf{a}}^{\mathrm{e}} \Psi_{\mathbf{b}}^{\mathrm{g}}\right\rangle$ to $\left|\Psi_{\mathbf{a}}^{\mathrm{g}} \Psi_{\mathbf{b}}^{\mathrm{e}}\right\rangle$ via interactions: $J_{\alpha}$ and $J_{\beta}$.

where $\mathbf{A}$ is the antisymmetrizer; $\Psi_{\mathbf{a}}^{\varepsilon}$ and $\Psi_{\mathbf{b}}^{\varepsilon} \quad(\varepsilon=\mathrm{g}$ or e) are the wave functions of molecules adjacent to each other. In the $a b$ plane, DNTT molecules are arranged in the herringbone pattern; a molecule in the unit cell has three inequivalent neighbors [Fig. 5(a)] and each pair of molecules has different electronic couplings. In what follows, we describe the electronic coupling constant of a pair $k(k=1,2$, or 3 ) as $J^{(k)}$. Because the absorption band of a hole is mainly contributed by the transition from SOMO to LUMO of a DNTT chromophore, the electronic configuration of the excited state of a hole is described as one-electron in LUMO and no-electron in HOMO [see Fig. 5(b)]. Thus $J^{(k)}$ is described as

$$
\begin{aligned}
J^{(k)} & =\left\langle\mathbf{A} \psi_{\mathbf{a}}^{\mathrm{L}}(1) \psi_{\mathbf{b}}^{\mathrm{H}}(2) \psi_{\mathbf{b}}^{\mathrm{H}}(3)\left|H_{e}\right| \mathbf{A} \psi_{\mathbf{a}}^{\mathrm{H}}(1) \psi_{\mathbf{a}}^{\mathrm{H}}(2) \psi_{\mathbf{b}}^{\mathrm{L}}(3)\right\rangle \\
& \propto J_{\alpha}^{(k)}-J_{\beta}^{(k)}, \\
J_{\alpha}^{(k)} & \equiv\left\langle\psi_{\mathbf{a}}^{\mathrm{L}}(1) \psi_{\mathbf{b}}^{\mathrm{H}}(2) \psi_{\mathbf{b}}^{\mathrm{H}}(3)\left|H_{e}\right| \psi_{\mathbf{a}}^{\mathrm{H}}(1) \psi_{\mathbf{a}}^{\mathrm{H}}(2) \psi_{\mathbf{b}}^{\mathrm{L}}(3)\right\rangle \\
J_{\beta}^{(k)} & \equiv\left\langle\psi_{\mathbf{a}}^{\mathrm{L}}(1) \psi_{\mathbf{b}}^{\mathrm{H}}(2) \psi_{\mathbf{b}}^{\mathrm{H}}(3)\left|H_{e}\right| \psi_{\mathbf{b}}^{\mathrm{L}}(1) \psi_{\mathbf{a}}^{\mathrm{H}}(2) \psi_{\mathbf{a}}^{\mathrm{H}}(3)\right\rangle,
\end{aligned}
$$

where $\psi_{\mathbf{n}}^{\mathrm{H}}(m)$ and $\psi_{\mathbf{n}}^{\mathrm{L}}(m)(\mathbf{n}=\mathbf{a}, \mathbf{b}, m=1,2,3)$ are the wave functions of HOMO and LUMO occupied by the $m$ th electron, respectively. The electronic coupling is composed of two terms [Fig. 5(b)]: (1) the first term $J_{\alpha}^{(k)}$ corresponds to a Förstertype energy transfer accompanied with one electron transfer between HOMOs of molecules at $\mathbf{a}$ and $\mathbf{b}$ of pair $k$. (2) The second term $J_{\beta}^{(k)}$ corresponds to a transfer of two electrons between HOMOs and the transfer of one electron between LUMOs through exchange interaction.

There are two major intermolecular electronic couplings between two chromophores: long-range Coulombic interaction [44] and short-range interaction including electronexchange interaction $[45,46]$. While the long-range Coulombic 
TABLE II. Transfer integrals of hole $\left(t_{\mathrm{h}}\right)$, electron $\left(t_{\mathrm{e}}\right)$, and the relative ratio of $t_{\mathrm{h}}^{2} t_{\mathrm{e}}$ for three different pairs in a DNTT crystal. The values of $t_{\mathrm{h}}$ and $t_{\mathrm{e}}$ are adapted from Ref. [43]. Note that the values of $t_{\mathrm{h}}$ are taken with reversed signs [50].

\begin{tabular}{lcccc}
\hline \hline & $k$ & $t_{\mathrm{h}}^{(k)}(\mathrm{meV})$ & $t_{\mathrm{e}}^{(k)}(\mathrm{meV})$ & ratio of $\left(t_{\mathrm{h}}^{(k)}\right)^{2} t_{\mathrm{e}}^{(k)}$ \\
\hline Pair 1 & 1 & -81 & -27 & 1 \\
Pair 2 & 2 & -28 & 37 & -0.164 \\
Pair 3 & 3 & 94 & -45 & 2.245 \\
\hline \hline
\end{tabular}

interaction dominates over the short-range interaction when the chromophores are located remotely, the short-range interaction strength dramatically increases as the orbitals of chromophores start to overlap. For example, in the case of naphthalene, when the intermolecular distance is in the range of $3 \sim 6 \AA$, the short-range term dominates over the Coulombic interaction [46]. The distance between sulfur atoms of nearest neighbor molecules in the DNTT crystal is $~ 3.6 \AA$ [43]. At this distance, the Coulombic interaction primarily due to dipole-dipole coupling is estimated to be merely $1 \mathrm{meV}$. Thus we assume that $\left|J_{\beta}^{(k)}\right|$ originating in the short-range interaction is much larger than $\left|J_{\alpha}^{(k)}\right|$ originating in the longrange Coulombic interaction.

Following the treatment by Closs et al. [47-49], we assume that the rate of electron and energy transfer can be expressed as a product of the rates of hole transfer (HT) between HOMOs and electron transfer (ET) between LUMOs. Because $J_{\beta}^{(k)}$ includes one ET and two HT processes, this term can be expressed as

$$
J_{\beta}^{(k)} \propto\left(t_{\mathrm{h}}^{(k)}\right)^{2} t_{\mathrm{e}}^{(k)}
$$

where $t_{\mathrm{h}}^{(k)}$ and $t_{\mathrm{e}}^{(k)}$ are the transfer integrals of holes and electrons between adjacent molecules of pair $k$, respectively. The transfer integrals in HT and ET processes calculated by Sánchez-Carrera et al. [43] are shown in Table II.

\section{G. Comparison with experimental results}

Referencing the calculated values of $t_{\mathrm{h}}^{(k)}$ and $t_{\mathrm{e}}^{(k)}$ in Table II, we simulated CM spectra as a function of transfer integrals. Because the electronic coupling term $J_{\beta}^{(k)}$ more strongly depends on the hole transfer integral $t_{\mathrm{h}}^{(k)}$ as in Eq. (8), we fixed $t_{\mathrm{e}}^{(k)}$ of three different pairs of molecules at the values given in Table II and examined how the spectral features are varied with $t_{\mathrm{h}}^{(k)}$. Here, the relative strengths of hole transfer integrals among three different pairs were kept as in Table II: $t_{\mathrm{h}}^{(1)}=$ $-0.081 t, t_{\mathrm{h}}^{(2)}=-0.028 t$, and $t_{\mathrm{h}}^{(3)}=0.094 t$; thereby, $J_{\beta}^{(1)}=$ $0.1 t^{2}, J_{\beta}^{(2)}=-0.0164 t^{2}$, and $J_{\beta}^{(3)}=0.2245 t^{2}$, where $t$ is a free parameter. Figure 6 shows the simulated spectra as a function of $t$ from 0 to 1.1 . The spectrum at $t=0$ reproduces that of DNTT cation, showing a definite vibrational progression. As $t$, hence $t_{\mathrm{h}}$ increases, the vibrational progression is blurred. In addition, the intensity at the lower absorption edge decreases, while the peak is shifted to the higher energy. At $t \geqslant 0.8$, the vibrational progression is completely lost and the spectra show typical $\mathrm{H}$-aggregate characteristics [39]. While the whole band

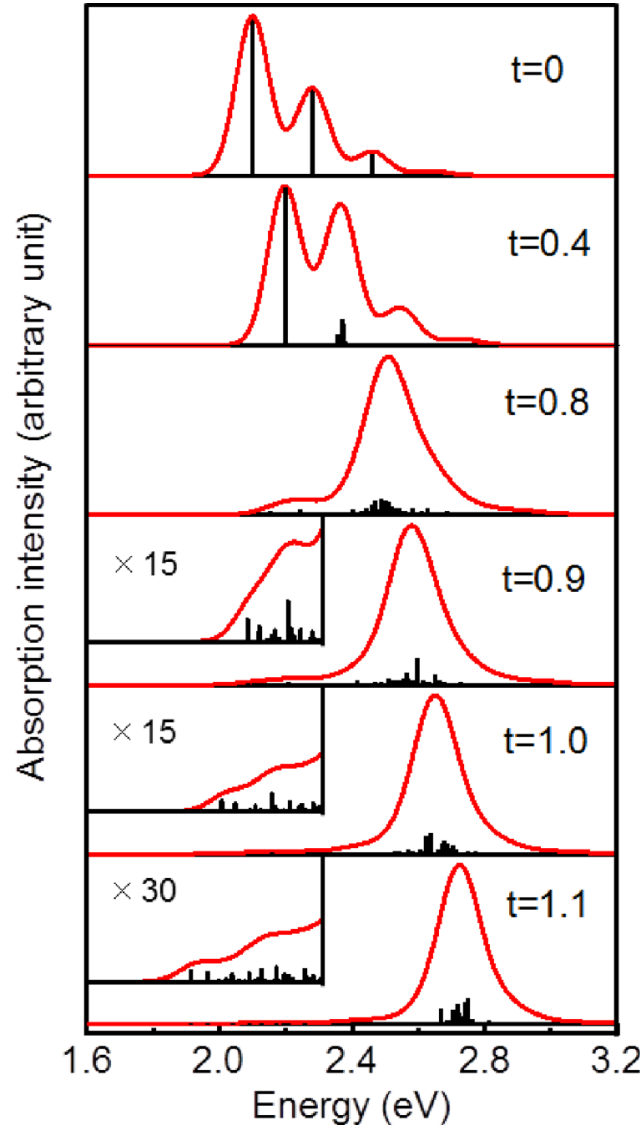

FIG. 6. (Color online) Normalized simulated absorption spectra of holes in DNTT as a function of $t$, while the relative strengths in the electronic couplings of three different pairs are fixed as $J_{\beta}^{(1)}=0.1 t^{2}, J_{\beta}^{(2)}=-0.0164 t^{2}$, and $J_{\beta}^{(3)}=0.2245 t^{2}$. The hole transfer integrals are varied as $t_{\mathrm{h}}^{(1)}=-0.081 t, t_{\mathrm{h}}^{(2)}=-0.028 t$, and $t_{\mathrm{h}}^{(3)}=$ $0.094 t$ from $t=0$ to 1.1 . The height of a stick indicates a spectral weight of each transition and the red curves are the convolution of stick spectra with a Gaussian line shape, $\Gamma(E)=\exp \left(-E^{2} / \gamma^{2}\right)$ with $\gamma=0.005 \mathrm{eV}$. Insets show the magnified spectra in the low-energy region.

structure shows blue shifts with increasing $t$, the absorption band edge is stretched to the lower energy.

Note that these changes in the spectral features of simulated spectra reproduce qualitatively the $n$ dependence of the $\mathrm{CM}$ spectra of $\mathrm{C}_{n}$-DNTT observed (Fig. 4). Figure 7 shows comparison between the observed $\mathrm{CM}$ spectra and the simulated lowest band. The redshifts observed in $\mathrm{C}_{n}$-DNTT TFTs with $n=8,10$, and 12 can be well reproduced by the simulation with $t=1.04,1.06$, and 1.08 , respectively. Namely, the $n$ dependence of the absorption band edge observed indicates that the hole transfer rate increases with increasing alkyl chain length. Therefore the analysis of the CM spectra confirms the fastener effect in the alkylated DNTTs; the alkyl chains change the mutual orientations of DNTT chromophores so as to enhance the intermolecular overlap of $\pi$ orbitals. This is consistent with crystal structural analysis with $\mathrm{x}$-ray diffraction; it has been demonstrated that introducing long alkyl chains at both ends of DNTT reduces interchromophore misalignment along $c$-axis and shortens the interchromophore 


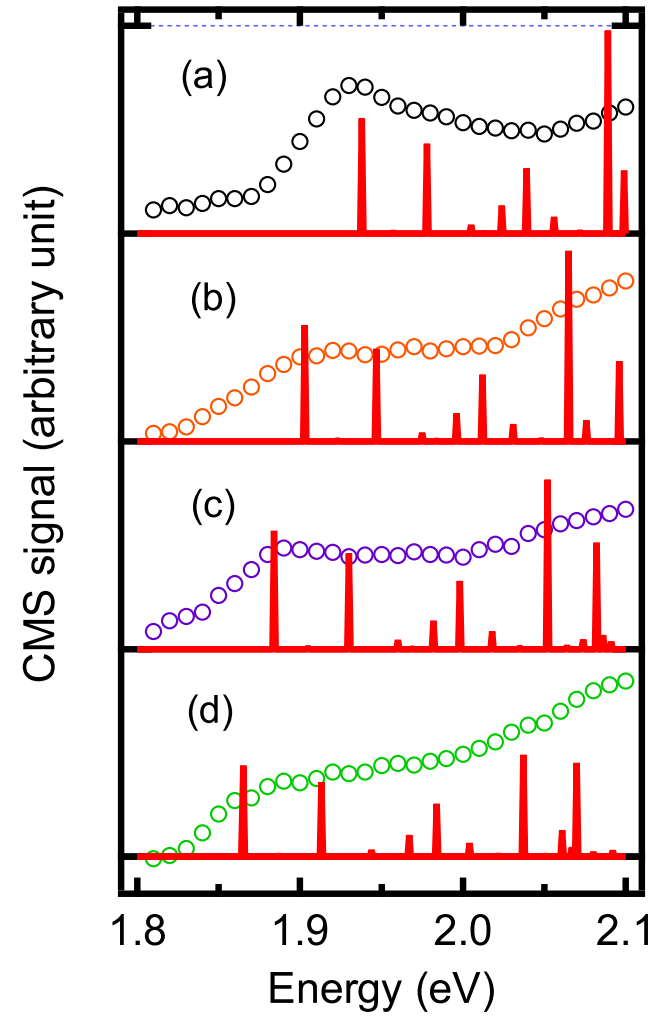

FIG. 7. (Color online) Comparison between observed CM spectra (open circles) and simulated absorption bands (sticks). CM spectra of (a) $n=0$, (b) 8, (c) 10, and (d) 12 and simulations with (a) $t=1.00$, (b) 1.04 , (c) 1.06 , and (d) 1.08 . The whole simulated bands are shifted by a solution-to-crystal shift of $-0.07 \mathrm{eV}$.

distance [33]. Thus the structural changes improve the overlap of $\pi$ orbitals of adjacent molecules (see Fig. S8 in Ref. [36]).

The improvement in hole mobility evaluated from the $I-V$ curves, however, cannot be explained solely by the enhancement in the intermolecular coupling due to alkyl chains introduced. From the relative strengths of hole transfer integrals obtained from the spectral analysis, we can examine the $n$ dependence of hole mobility among the devices; the transfer integrals increase with the alkyl-chain length monotonically by a factor of 1.08 from $n=0$ to 12 . The Holstein polaron model, for example, predicts that the carrier mobility increases with $t_{\mathrm{h}}^{2}$; thus, only $17 \%$ of enhancement is accounted for by this model. In contrast, the hole mobilities of $\mathrm{C}_{n}$-DNTT evaluated from the $I-V$ characteristics (Table I) increases by a factor of 4 from $n=0$ to 10 , but the mobility decreases when the longer alkyl chains $(n=12)$ are introduced. Thus the relative transfer integrals deduced from the absorption band edges substantially underestimate the increase in hole mobility when the chain length is increased from $n=0$ to 10 . Moreover, we expect that the hole mobility increases monotonically up to $n=12$, because the transfer integral does not decrease at $n=12$. The discrepancies in the $n$-dependence of carrier mobility between the two independent measurements performed with the same devices suggest that the carrier mobilities estimated from the $I-V$ characteristics are strongly influenced by the mesoscopic morphologies of grains, the structure of grain boundaries, defects in grains, etc., whereas the increase in microscopic
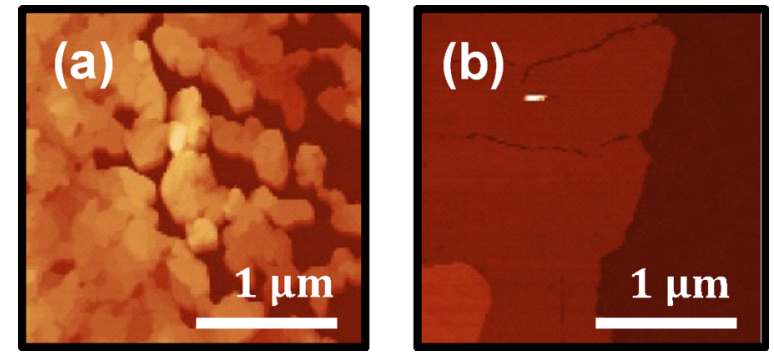

FIG. 8. (Color online) Typical AFM images of (a) DNTT and (b) $\mathrm{C}_{10}$-DNTT thin films with about monolayer thickness.

intrinsic hole transfer integral plays a minor role. The decrease in hole mobility associated with the elongation of chains from $n=10$ to 12 is likely attributed to the increase in the number of defects originating in the formation of gauche defects in the thin film of $\mathrm{C}_{12}$-DNTT.

The morphologies of thin films with a nearly monolayer thickness observed with AFM shown in Fig. 8 are suggestive in this regard. In the DNTT thin film, grains and boundaries between them are clearly visible. In contrast, the AFM image of the $\mathrm{C}_{10}$-DNTT thin film is highly smooth and almost continuously connected in a wider range. The average area of the grains in the $\mathrm{C}_{10}$-DNTT thin film is much larger than that in the DNTT thin film: 0.3 and $3.6 \mu \mathrm{m}^{2}$ for DNTT and $\mathrm{C}_{10}$-DNTT thin films, respectively (see Fig. S9 in Ref. [36]). This implies that the number of grain boundaries and barrier heights for intergrain hole transfer in the channel region are smaller in the $\mathrm{C}_{10}$-DNTT thin film than the DNTT film. These observations are also consistent with recent ESR studies; the barriers of intergrain hopping were estimated to be $45 \mathrm{meV}$ for DNTT [16] and $\sim 10 \mathrm{meV}$ for $\mathrm{C}_{10}$-DNTT [17]. Hence it is very likely that the mesoscopic morphology of thin films is more crucial to hole mobility than the microscopic fastener effect that manifests itself in increasing hole transfer integrals with the alkyl-chain length.

\section{CONCLUSIONS}

We have carried out systematic hole mobility measurements based on $I-V$ characteristics and the measurements of $\mathrm{CM}$ spectra of DNTT and $\mathrm{C}_{n}$-DNTT $(n=8,10$, and 12) as a function of alkyl-chain length using the same devices, together with measurements of the absorption spectra of cations of these compounds in a solution. The hole mobility increases by a factor of four as the alkyl-chain length is increased from $n=0$ to 10 , but decreases at $n=12$. The spectral features at the band edges of CM spectra systematically change with the alkyl-chain length: (1) the remaining vibrational progression in the absorption spectra of DNTT is completely lost in $\mathrm{C}_{n}$-DNTT ( $n=8,10$, and 12). (2) The band edge shifts to the lower energy with increasing alkyl-chain length. Simulations of absorption spectra of holes with the extended theory with Holstein-type Hamiltonians indicate that the hole transfer integral increases with increasing alkyl-chain length. Systematic measurements of CM spectra in combination with theoretical simulations prove that information on the hole transfer integrals can be extracted from the spectral features of $\mathrm{CM}$ spectra at least semiquantitatively. The analysis of $\mathrm{CM}$ 
spectra confirms the fastener effect at the microscopic level; the subtle changes in mutual orientations of DNTT chromophores by introducing alkyl chains enhance the intermolecular electronic coupling. However, the enhancement in hole transfer integrals by introducing alkyl chains cannot account for the substantial increase of hole mobility evaluated from $I-V$ curves. This indicates that the mesoscopic morphology of grains in the thin films plays a dominant role in the hole mobility of $\mathrm{C}_{n}$-DNTT TFTs over the microscopic arrangements of molecules.

\section{ACKNOWLEDGMENTS}

We thank Kazuo Takimiya, Meyong Jin Kang, Hirokazu Kuwabara, and Masahiro Hamada for providing us DNTT samples used in this work and Kazumoto Miwa and Naoko Namba for their help in fabricating the devices. This work was supported by the Grant-in-Aid for Scientific Research (A) from the Japanese Society for the Promotion of Sciences (Grant No. 22245001), Kyoto University Global COE program, and the program of Network of Joint Research Center for Advanced Materials and Devices.
[1] S. R. Forrest, Nature (London) 428, 911 (2004).

[2] C. Dimitrakopoulos and P. Malenfant, Adv. Mater. 14, 99 (2002).

[3] G. Horowitz, J. Mater. Res. 19, 1946 (2004).

[4] T. B. Singh and N. S. Sariciftci, Annu. Rev. Mater. Res. 36, 199 (2006).

[5] V. Coropceanu, J. Cornil, D. A. da Silva Filho, Y. Olivier, R. Silbey, and J.-L. Brédas, Chem. Rev. 107, 926 (2007).

[6] A. R. Murphy and J. M. J. Fréchet, Chem. Rev. 107, 1066 (2007).

[7] J. E. Anthony, Chem. Rev. 106, 5028 (2006).

[8] H. Inokuchi, K. Imaeda, T. Enoki, T. Mori, Y. Maruyama, G. Saito, N. Okada, H. Yamochi, K. Seki, Y. Higuchi, and N. Yasuoka, Nature (London) 329, 39 (1987).

[9] J. E. Anthony, J. S. Brooks, D. L. Eaton, and S. R. Parkin, J. Am. Chem. Soc. 123, 9482 (2001).

[10] C. Cui, W.-Y. Wong, and Y. Li, Energy Environ. Sci. 7, 2276 (2014).

[11] T. Lei, J.-Y. Wang, and J. Pei, Chem. Mater. 26, 594 (2014).

[12] S. F. Nelson, Y.-Y. Lin, D. J. Gundlach, and T. N. Jackson, Appl. Phys. Lett. 72, 1854 (1998).

[13] K. Marumoto, S. I. Kuroda, T. Takenobu, and Y. Iwasa, Phys. Rev. Lett. 97, 256603 (2006).

[14] H. Matsui, T. Hasegawa, Y. Tokura, M. Hiraoka, and T. Yamada, Phys. Rev. Lett. 100, 126601 (2008).

[15] H. Tanaka, M. Kozuka, S.-I. Watanabe, H. Ito, Y. Shimoi, K. Takimiya, and S.-I. Kuroda, Phys. Rev. B 84, 081306 (2011).

[16] H. Matsui, D. Kumaki, E. Takahashi, K. Takimiya, S. Tokito, and T. Hasegawa, Phys. Rev. B 85, 035308 (2012).

[17] Y. Kinoshita, H. Tanaka, Y. Shimoi, K. Takimiya, and S. Kuroda, Appl. Phys. Lett. 105, 033301 (2014).

[18] M. Harrison, R. Friend, F. Garnier, and A. Yassar, Synth. Met. 67, 215 (1994).

[19] M. Harrison, D. Fichou, F. Gamier, and A. Yassar, Opt. Mater. 9, 53 (1998).

[20] H. Sirringhaus, P. J. Brown, R. H. Friend, M. M. Nielsen, K. Bechgaard, B. M. W. Langeveld-Voss, A. J. H. Spiering, R. A. J. Janssen, E. W. Meijer, P. Herwig, and D. M. de Leeuw, Nature (London) 401, 685 (1999).

[21] P. J. Brown, H. Sirringhaus, M. Harrison, M. Shkunov, and R. H. Friend, Phys. Rev. B 63, 125204 (2001).

[22] Y. Y. Deng and H. Sirringhaus, Phys. Rev. B 72, 045207 (2005).
[23] S. Haas, H. Matsui, and T. Hasegawa, Phys. Rev. B 82, 161301 (2010).

[24] Y. Harima, Y. Ishiguro, K. Komaguchi, I. Imae, and Y. Ooyama, Chem. Phys. Lett. 495, 228 (2010).

[25] T. Sakanoue and H. Sirringhaus, Nat. Mater. 9, 736 (2010).

[26] J.-F. Chang, T. Sakanoue, Y. Olivier, T. Uemura, M.-B. DufourgMadec, S. G. Yeates, J. Cornil, J. Takeya, A. Troisi, and H. Sirringhaus, Phys. Rev. Lett. 107, 066601 (2011).

[27] T. Holstein, Ann. Phys. 8, 325 (1959).

[28] H. Yamagata, J. Norton, E. Hontz, Y. Olivier, D. Beljonne, J. L. Bredas, R. J. Silbey, and F. C. Spano, J. Chem. Phys. 134, 204703 (2011).

[29] F. C. Spano and H. Yamagata, J. Phys. Chem. B 115, 5133 (2011).

[30] C. M. Pochas and F. C. Spano, J. Chem. Phys. 140, 244902 (2014).

[31] T. Yamamoto and K. Takimiya, J. Am. Chem. Soc. 129, 2224 (2007).

[32] M. J. Kang, I. Doi, H. Mori, E. Miyazaki, K. Takimiya, M. Ikeda, and H. Kuwabara, Adv. Mater. 23, 1222 (2011).

[33] K. Nakayama, Y. Hirose, J. Soeda, M. Yoshizumi, T. Uemura, M. Uno, W. Li, M. J. Kang, M. Yamagishi, Y. Okada, E. Miyazaki, Y. Nakazawa, A. Nakao, K. Takimiya, and J. Takeya, Adv. Mater. 23, 1626 (2011).

[34] GAUSSIAN09 Revision A.1, Gaussian Inc., Wallingford, CT, 2009.

[35] Y. Ishino, K. Miyata, T. Sugimoto, K. Watanabe, Y. Matsumoto, T. Uemura, and J. Takeya, Phys. Chem. Chem. Phys. 16, 7501 (2014).

[36] See Supplemental Material at http://link.aps.org/supplemental/ 10.1103/PhysRevB.91.195306 for the raw data of $I-V$ characteristics, the gate-voltage dependence of CM spectra, the absorption spectra of neutral thin films, the crystalline structures and grain size distributions of DNTT and $\mathrm{C}_{10}$-DNTT, and the theoretical supplements for spectral simulations.

[37] M. Hoffmann and Z. G. Soos, Phys. Rev. B 66, 024305 (2002).

[38] J. Roden, G. Schulz, A. Eisfeld, and J. Briggs, J. Chem. Phys. 131, 044909 (2009).

[39] F. C. Spano, Acc. Chem. Res. 43, 429 (2010).

[40] A. Stradomska and P. Petelenz, J. Chem. Phys. 130, 094705 (2009).

[41] M. R. Philpott, J. Chem. Phys. 55, 2039 (1971).

[42] F. C. Spano, J. Chem. Phys. 116, 5877 (2002).

[43] R. S. Sánchez-Carrera, S. Atahan, J. Schrier, and A. AspuruGuzik, J. Phys. Chem. C 114, 2334 (2010). 
[44] T. Förster, Discuss. Faraday Soc. 27, 7 (1959).

[45] D. L. Dexter, J. Chem. Phys. 21, 836 (1953).

[46] G. D. Scholes and K. P. Ghiggino, J. Phys. Chem. 98, 4580 (1994).

[47] G. L. Closs, L. T. Calcaterra, N. J. Green, K. W. Penfield, and J. R. Miller, J. Phys. Chem. 90, 3673 (1986).
[48] G. L. Closs, P. Piotrowiak, J. M. MacInnis, and G. R. Fleming, J. Am. Chem. Soc. 110, 2652 (1988).

[49] G. L. Closs, M. D. Johnson, J. R. Miller, and P. Piotrowiak, J. Am. Chem. Soc. 111, 3751 (1989).

[50] K. Senthilkumar, F. C. Grozema, F. M. Bickelhaupt, and L. D. A. Siebbeles, J. Chem. Phys. 119, 9809 (2003). 